\title{
Simulation-based approach for paper folding with the aim to design the origami-performing robotic system
}

\author{
Phuong Thao THAI*, Maria SAVCHENKO*, Hoan Thai Tat NGUYEN** and Ichiro HAGIWARA* \\ *Graduate School of Advanced Mathematical Sciences, Meiji University \\ 4-21-1 Nakano, Nakano-ku, Tokyo 164-8525, Japan \\ E-mail: thaithao@meiji.ac.jp \\ ${ }^{* *}$ School of Mechanical Engineering, Hanoi University of Science and Technology \\ 1 Dai Co Viet street, Hanoi, Viet Nam
}

Received 25 November 2015

\begin{abstract}
Origami is a traditional Japanese craft that is based on the folding of the designed structure and can be widely used by industry. Origami folding is not a difficult task for human hands; however, folding by robot hands is such a challenge. In this paper, we suggest a novel approach for designing the origami-performing robotic systems. The main idea of the proposed method is to simulate the forming crease lines in origami models and folding behavior of a sheet of paper by robot end-effectors. The simulation approach becomes the main option in the real-world related with a robotic activity. Our investigation results show that many design parameters of a robot such as geometrical and topological, shape and configuration of the robotic arms, type of forces and their distributions, and others can be defined by the simulation of the folding origami structures. It means that the results of the simulation can be used as a basis of the final robot design without a series of experimental tests. Problems of the numerical simulation, including paper material structure, simulation origami model, the distribution and values of the applying forces, and others are considered carefully. The results of the design process of the robotic system based on the simulation of origami crease forming confirm our idea to use simulation approach with LS-DYNA solver to build the origami-performing robot for complex origami structures. The origami model "Star" was taken as an example to demonstrate the method for the different types of the creases in origami.
\end{abstract}

Key words : Design, Structural analysis, Structural design, Robot arm, Manipulator

\section{Introduction}

Nowadays, robots are used everywhere in everyday life. With all the recent advances in robotics, practical robots become available for home use. These types of robots have to be portable with the small gabarits and weights to do some real work at home or at the company offices to exclude the humans from making the target work. The papercraft production as an art and a hobby for many people, including children, is the important part of the human activity and can become a popular application for the origami-performing robot. The aim of the origami-performing robot is to exclude the humans from the complex and time-consuming operations in producing the origami models. Papercraft, CAD or origami design software can be connected to the robot system. Users will receive possibility to create own origami structures, which will be produced at home directly by a robot.

The geometrical sizes of the compact robot create possibility to use a robot in limited spaces, such as home room (for instance, on a desk), children facilities, offices and so on, for the production of paper models.

Origami (paper folding) is a traditional art of Japan for producing the 3D paper models from the flat sheets of paper. Nowadays everyone can create many $3 \mathrm{D}$ origami forms from the various patterns. Using robotics for folding the $2 \mathrm{D}$ 
paper pattern for forming the $3 \mathrm{D}$ origami model is considered in this paper. Manufacturing origami-inspired products requires robots capable of bending and folding materials.

Using robotics for folding the paper origami models is not a brand new idea. During the last ten years several realizations of this idea has been suggested in some publications (from Reference section). The robot design included a table, a blade, a clamp, a plate attached to a robot arm is demonstrated (Balkcom and Mason, 2004). This system was succeeded in folding some models like samurai hat, air plane. Folding origami by two robotic hands was suggested in the paper by Tanaka et al. (2007). The authors concentrated on origami patterns, which contain various typical folds and do not include many folding steps. They chose the "tadpole" origami model for their target. However, this system did not succeed in making the sharp creases on the folding patterns: sheets can slip out from the fingertip during the folding operation. Another approach that is connected to packaging robot is presented (Yao et al., 2010); nevertheless, this approach does not focus on how to make the crease lines on the sheet of paper. They just concentrated on forming the 3D shapes with some folding techniques, but do not use the imitating movement of human hands or fingers. In their work, the folding systems were designed before and the folding patterns were modified to fit the manipulating structure. Two robot hands are used for paper folding in the paper by Elbrechter et al. (2012). They apply a method for real-time detection and physical modeling of paper and suggest an approach to recognize the shape of a sheet of paper. A new robotic origami system is proposed (Namiki and Yokosawa, 2015). System configuration includes the left and right hands with two fingers. To produce dexterous paper folding the authors extract some dynamic motion primitives, which contain visual or force information. Integrating dynamic primitives that consists of sensory feedback control allows paper folding. Simulation of the deformation of a paper sheet is developed with modeling of a sheet of paper by triangular mesh. All above systems were designed based on an experimental approach with the existing robotic system to fold the origami sheets.

To our knowledge, there are no publications connected to the simulation-based design for the origami-performing robotic system. In this paper we introduce our vision of the designing the origami-performing robot. In our current research, we decided to consider the relationship between the design process for a robot and a simulation of the forming the crease lines on origami structures by the robotic arms. We suppose that each origami folding operation can be considered as a function of the mechanical systems such as a robot. Robot arm motion can be simulated for performing the folding operations. In the current simulation stage, we use only end-effectors.

The main contributions in this paper are: simulation methodology of the forming the accurate creases in a sheet of paper; design of the robot end-effectors and defining their positions on a sheet of paper; calculation of the force values and determining the directions of their applications; a sequence decision for the folding operations for the robotic arm planning. As we demonstrate by our results, the simulation-based approach can be considered as the basis for a design of the realistic origami-performing robot.

Simulation results show the possibility of using robots for conducting the task in origami processing as an analogy with the human fingers manipulations. The design based on experiment only is difficult, expensive, time-consuming and so on. The simulation approach based on software becomes the main option in the real-world related with a robotic activity for creating basis for their hardware design. The main problem that we try to solve is how to form the crease lines in the simulation model. In related works mentioned above, the crease making approach was considered in the different ways. The crease lines are produced by putting a sheet of paper in a slot and formed by using the clamp shut (Balkcom and Mason, 2004). In research by Tanaka et al. (2007), a rubber ball slides on the paper to form the crease after folding by moving a fingernail attached to the fingertip on an arm-manipulator. In the paper by Yao et al. (2010), the object is carton box with paperboard material. Creases are made before bending panel to form the box shape. Although these above methods are able to form the crease lines on the origami patterns, the accuracy of the results was not analyzed and illustrated clearly. We use paper material (hereinafter we call it paper) in the simulation model for making apparent crease lines during the paper folding process. In this case, it is necessary to investigate a structure of paper and mechanical operations with it for creating the crease lines by the simulation process. Additionally, the magnitudes of the force and directions of their application in simulation are considered carefully to achieve the best results for creasing.

In our simulation, the creases appear when bending paper around the folding lines according to the origami pattern. The 3D structural simulation of the origami is carried out by the finite element modeling in LS-DYNA software Livermore Software Technology Corporation (2001). We succeeded in the numerical simulation by using finite element method (FEM), and the design of the robot and its behavior during the creases forming are illustrated and optimized by 
using the simulation software LS-DYNA.

The following is a brief overview of this paper: Section 2 introduces the approach for design of robotic system for origami application, with the schematic design sketching, system configuration in details, and geometrical shapes for folding fingers. In section 3 , the simulation of positions and contacts between paper and holding fingers is carried out to consider problems during a bending process and their solutions. In section 4, to investigate folding conditions completely, we consider the paper structure and method of making the crease line appearance on paper, including position for robotic arms and folding forces. In section 5, the proposed method is experimentally applied for folding the pattern of the origami model to illustrate the effectiveness of the proposed folding approach. Results and limitations of research are discussed and summarized in section 6. In the last section, main conclusions and proposals for future research are presented.

\section{An approach for the design of a robotic system for origami applications 2.1 Schematic Design Sketching}

The schematic design is the first phase of the research and presents the conceptual design of a robot hardware. As mentioned in Introduction, our final target is to design the origami-performing robot. It means that the first criterion is to design the individualized robot. With this goal, our robot is required to be light-weight, portable, and be easy to manage the applications. The second criterion is connected to the robot manipulations. Robot-manipulator consists of the arms with end-effectors (fingers) and body. For designing the conceptual model of a robot and its manipulation it is necessary to observe how human hands operate during the folding process of a sheet of paper. Human being can apply some tricks to make the crease lines during the folding process. Human fingers grasp two corners of the origami paper pattern and bring them into precise alignment. Then the fingers of one hand smooth the bulges on a sheet of paper. Since paper does not stretch, the creasing lines form the extreme region of paper along the diagonal directions. Crease lines are sharpened by applying strength using fingertips. This operation is important for folding an origami pattern by the robot arms. It seems to be a simple problem when a person folds a sheet of paper; anyway, there are at least 6 states in process: holding, folding, flattening (creasing), flipping, gluing, and checking. Automation of a process of the deformation of the flexible objects by using a robot is the difficult task for an experimental approach. The simulation based on computer software becomes the main option in real-world robotic related activity.

From studies and requirements mentioned above, we suppose that the conceptual model of the developed robot system includes a working table and two arms: one folding and one holding (Fig. 1). For the folding arm, the main operation is a paper folding. The holding arm is used to fix paper on the working table. The experimental folding machine presented by Balkcom and Mason (2004) includes a blade press for forming the creases and a working table. Our suggested conceptual design is closed to the design described by Tanaka et al. (2007) for the "tadpole" model. The authors of this paper use a rubber ball to form creases and explain that their robot design should be redesigned for each origami model. In our case, we try to design the robotic system that will be not redesigned for the different origami structures. Our purpose is to design the robotic system that can fold various kinds of origami models; therefore our design is more complicated to satisfy that requirement.

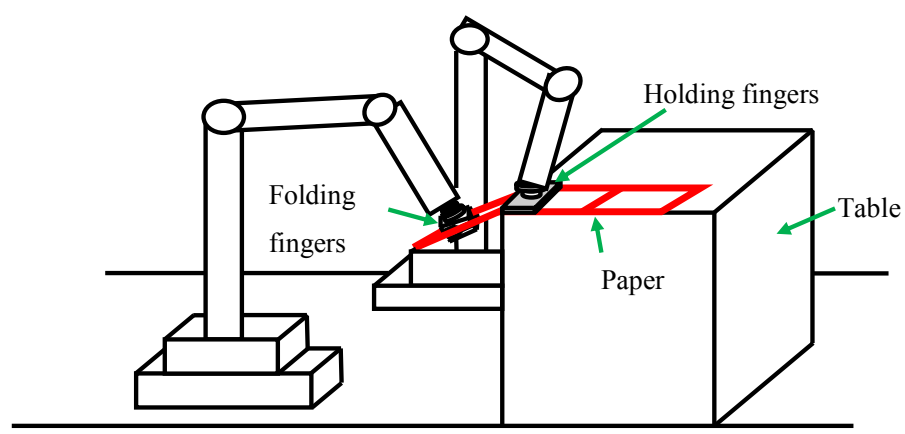

Fig. 1. Sketch of the conceptual robot design. 


\subsection{Detail robot configuration for the schematic design}

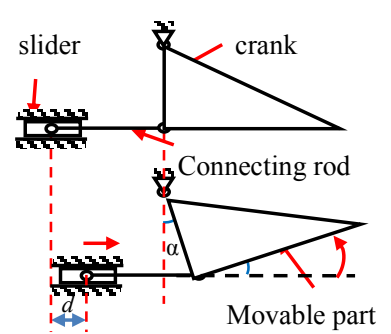

a. Gripper mechanism

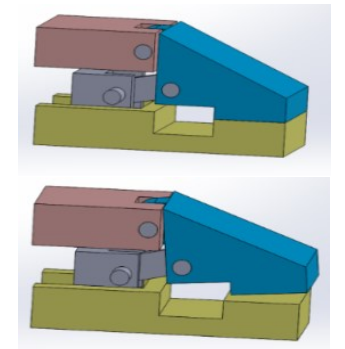

b. CAD gripper design

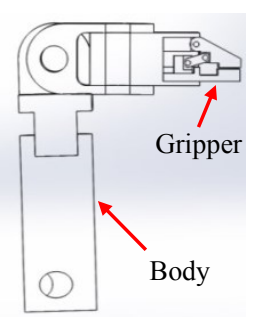

c. Cross-section of the robot arm

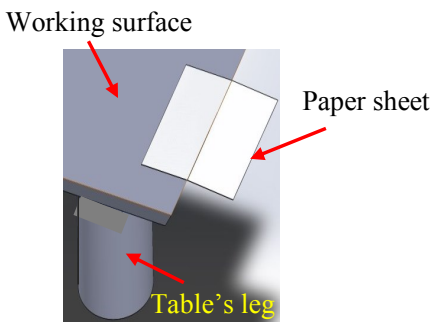

d. Design of the working table

Fig. 2 The components of the robot design.

The object of our robotic system is A4 format of the sheets of paper. In this case, the design of the robot should guarantee the satisfaction to this requirement.

In our system, both folding and holding arms with the different functions have the same configuration: 3 revolute joints for each manipulator (Fig. 1). End-effectors are the most important part of each robot arm. We call holding arm's end-effector as the holder (holding finger) and folding arm's end-effector as the gripper (folding finger). Grasping a paper sheet is classified as a lateral grasping. A human generally uses only two fingers: the thumb and the forefinger supported by others for the lateral grasping. The thumb is used to apply force to the objects supported by the forefinger. Grip force is required less for grasping a flat object like a sheet of paper. The gripper includes at least 2 fingers: a thumb and a forefinger. This gripper has to grasp one side of paper and bend it around a crease line. The mechanism, which we choose for the folding gripper, is the crab's propodus. There are two fingers in the crab's propodus: one fixed finger and one movable finger. The fixed finger produces the movement of dactyl (the movable finger) by two muscles: muscle attaching on the flexor apodeme for closing and muscle attaching on the extensor apodeme for gaping. The advantages of this structure are: the simple lever and mechanism, an adaptation to particular ways of feeding. Hence, we develop this mechanism to build the folding gripper with a fixed finger and a movable one (Fig. 2a). There are 4 parts in the mechanism like a "crank-slider": a base, crank, slider, and connecting rod. The base is fixed, the crank is rotated around the fixed axis, the slider motion is a horizontal translation and the connecting rod movement is a motion in the plane. When the slider moves to the right, it transfers the motion to the connecting rod and makes the crank rotation (open up to grasp paper).

For the characteristic of our system, the gripper should be open and closed as quickly as possible. As paper is not very thick, the opening angle of the gripper is not unnecessarily to be big. The opening angle can be calculated as below:

$$
\sin \alpha=\frac{d}{l},
$$

where $d$ is slider's moving distance and $l$ is crank's length (Fig. 2a). Moving distance $d$ is connected to the paper thickness $h$. In this case, minimal value of $\alpha$ should be defined by $h$ : $\operatorname{minimum}(\alpha)=\arcsin (h / l)$.

The geometrical shape for the grippers is inspired by human actions for folding a sheet of paper. The shape of the gripper from computer-aided design (CAD) and the cross-section of the grippers are demonstrated in Fig. $2 \mathrm{~b}$ and Fig. 2c. The design of the holding arm is the same as the folding one. In this case, the shapes of the holders are rectangular cuboids.

To make a perfect crease, one side of a sheet of paper is fixed on the working table while the grippers grip the free side of the sheet for bending it around the crease line. The purpose of this step of investigation is to make the crease lines appearance obviously after bending. A sheet of paper has infinite degrees of freedom; it is better to fix it along the crease line in order to make paper constraint and prevent bubbling and wrinkling. Since the crease lines may have different lengths, the design of the holder with only one part in a constraint length will not be an optimal choice. Also, 
this design will not satisfy the requirement of the light-weight and portable robot. Therefore, our idea is to design the holding arm with 2 holders that can fix a sheet of paper on the working table. The best positions for the grippers on the sheet of paper should be found during the simulation. To simplify the robot's mechanism (in comparison with the actions of the human hands, when a sharpness of the crease line is produced by the additional operations by applying the force from the human hand), the working table is designed with a sharp edge for accurate forming the crease lines (see Fig.2d).

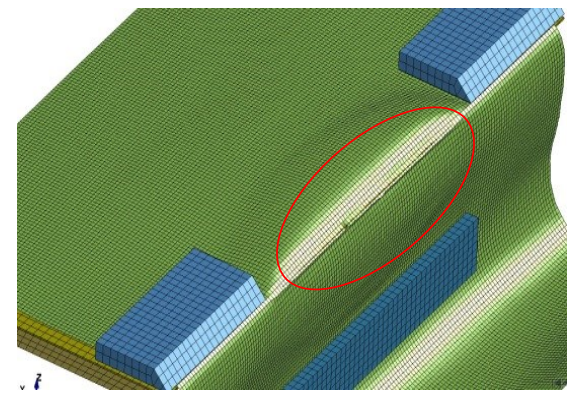

a. Two holders

Fig. 3 Bubbling problem.

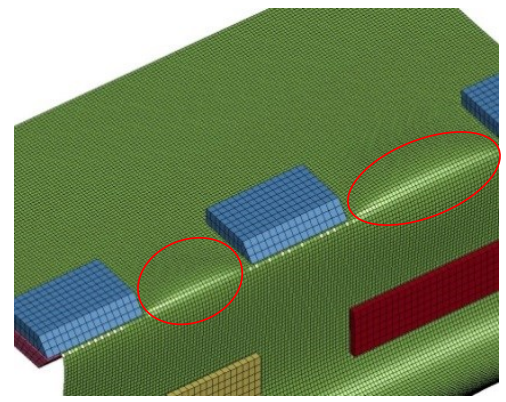

b. Three holders

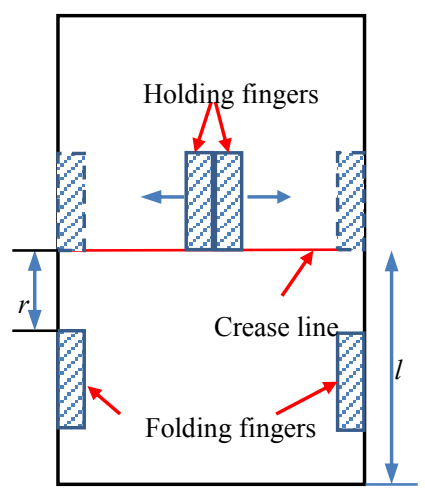

a. Sketch of arm finger's modification

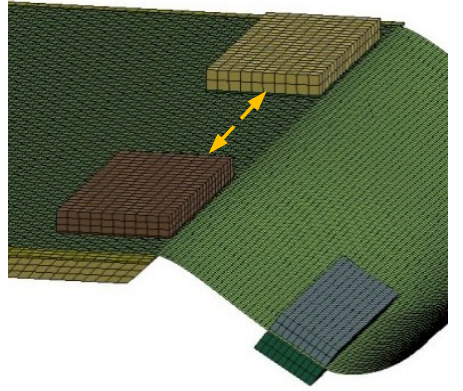

b. Simulation results without bubbling

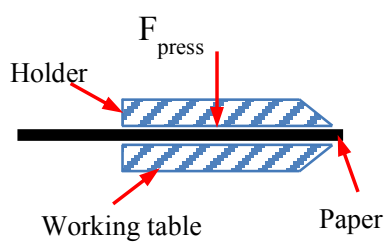

c. Pressing force

Fig. 4 Simulation solution for bubbling.

\section{Contact simulation between a paper sheet and the holders}

Two holders are put at the boundaries of the folding sheet of paper (Fig. 3a).

The friction of the holders is a problem in the folding simulation. As it is noticed in the paper by Namiki and Yokosawa (2015) the holders with a higher friction coefficient are desired. In our simulation, the friction between the contact portions of the holders and a sheet of paper is considered as the contact function between the surface areas in LS-DYNA solver. This type of the contact allows transmitting a tangential load if relative sliding occurs, when contact friction between the holder material and a sheet of paper is active. We use Polypropylene (PP) as a holder material. If the magnitude of a gripping force is big, the paper is likely to be broken. Additionally, during the folding process without stretching, paper is bubbling, creases cannot be formed. In the case of creating the origami model by humans, one finger is used to flatten the bubble on paper. By using three holders instead of 2 holders, we bend paper without stretching, however the accuracy of simulation still does not increase (Fig. 3b). Based on this simulation results, we decided to use the holders that can adjust the length of a crease line and translate along the crease line to flatten the bubbled sheet in the crease area (Fig. 4a). With this idea, the type of contact mentioned above does not work because in this case, the sheet of paper is significantly deformed. Therefore, we have to use another LS-DYNA contact function that allows translating the holders along a sheet of paper without a paper deformation. We choose TIEBREAK contact 
function because it transmits both compressive and tensile forces to the paper sheet from the holders. Therefore, it creates the stronger contact than the previous one. As the result, the sheet of paper is fixed on the working table, no bubbles occur during the folding process, and the holders can be designed with the smaller sizes (Fig. 4b). The holders are designed for moving along the crease line: from the center part of the crease area in both directions to the sheet's boundaries. To perform the translational movement the holders include two plates connecting with a screw. The screw moves to adjust the length of the crease lines according to the folding pattern. Finally, two grippers are located on the boundaries of the sheet under the pressing holder force (Fig. 4c). Based on the analysis of the contact between the holders and paper we assume that a magnitude of the pressing holder force should be enough to keep the sheet of paper on the working table.

\section{Folding conditions}

In this section, we consider the simulation conditions for a crease forming. The structure of paper material is discussed to understand forming the creases during the simulation folding process.

\subsection{Mechanical properties of paper}

Here, we need to discuss about a structure of paper and how to form creases on paper (Lister). Paper is made up of vegetable fibers, which are felted. The fibers are brittle and during the crease processing paper fibers are permanently fractured along the line of the crease. This is a permanent line of tiny fractures that forms the crease. Because this line is indelible, paper is said to "remember" the crease. Even hot ironing will not get rid of the crease. Finite Element Analysis (FEA) is used to approximate and verify component reaction under various loading conditions. Material is a main component to FEA. Elastic material property has a linear stress-strain relationship regardless of the load applied while the elastic-plastic has a linear relationship up to yield point and then becomes nonlinear beyond yield point. Fully elastic deformation is (Hookes Law): $\sigma=E \varepsilon$, where $\sigma$ is stress, $E$ is modulus of elasticity, $\varepsilon$ is strain.

Paper displays various unconventional mechanical properties both below and beyond the plastic limit, all the way up to its failure. As it was well known, there is approximately linear section at small strains in the load-elongation curve of paper. Nevertheless, the yield point has no unique definition because the deviation from the linear portion grows gradually as elongation increases. Thus, one way to define the yield point is to identify stress (and strain) at which the smooth curve begins to deviate from a straight line beyond a certain percentage.

The linear elastic, orthotropic behavior of paper prior to yielding can be modeled by the classical model.

$$
\begin{aligned}
& \sigma_{11}=\frac{E_{1}}{1-v_{12} v_{21}} \varepsilon_{11}+\frac{E_{2} v_{12}}{1-v_{12} v_{21}} \varepsilon_{22}, \\
& \sigma_{22}=\frac{E_{1} v_{21}}{1-v_{12} v_{21}} \varepsilon_{11}+\frac{E_{2}}{1-v_{12} v_{21}} \varepsilon_{22}, \\
& \tau_{12}=G \gamma_{12}=2 G \varepsilon_{12}
\end{aligned}
$$

where the engineering constants are: $E_{1}=$ Young's modulus in $x_{1}$ - direction (MD), $E_{2}=$ Young's modulus in $x_{2}$ direction $(\mathrm{CD}), v_{12}=\frac{-\varepsilon_{22}}{\varepsilon_{11}}$ is the Poisson's ratio for strain in $x_{2}$-direction $(\mathrm{CD})$ when paper is stressed in $x_{1}$ direction (MD) only, $v_{21}=\frac{-\varepsilon_{11}}{\varepsilon_{22}}$ is the Poisson's ratio for strain $x_{1}$-direction (MD) when paper is stressed in $x_{2}$ direction (CD) only, $G=$ shear modulus in $x_{1} x_{2}$ - plane (Fig. 5).

Here the same constant of proportionality between elastic module $E_{1}$ and $E_{2}$, Poisson's ratios $v_{12}$ and $v_{21}$ is required so as to ensure that the stiffness matrix is orthotropic:

$$
\frac{E_{1}}{E_{2}}=\frac{v_{12}}{v_{21}}
$$




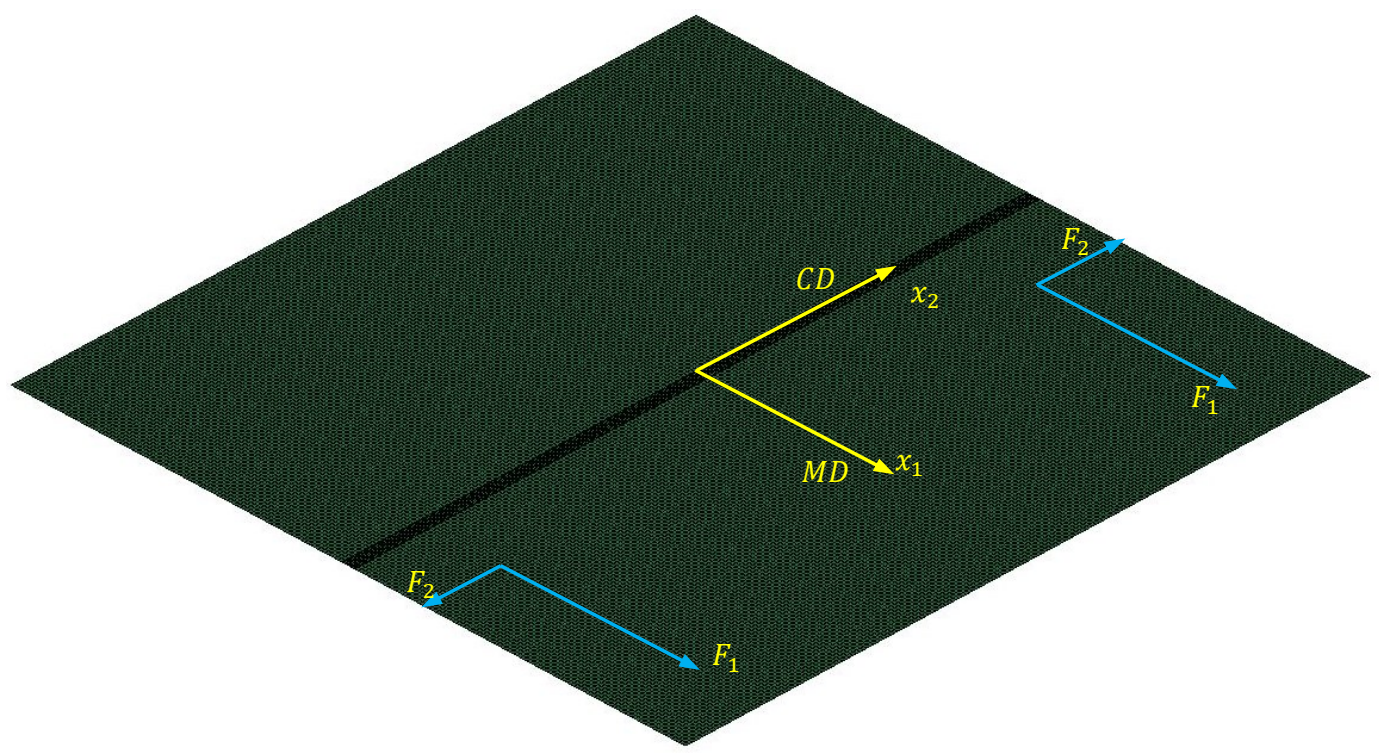

Fig.5 Paper model and force applications.

The crease line is a permanent deformation (shape changing) of a sheet of paper and can be considered as a plastic deformation. In the current simulation of the forming crease line tensile forces along MD direction and along CD direction have values equal to $10 \mathrm{~N}$. That configuration of forces allows us to apply the elastic analysis. The elasto-plasticity model is more complicated for the calculation but it is necessary to produce this analysis for the verification of the real quality of the formed creases.

In the simulation of the crease forming, we use the elasticity model in LS-DYNA. Nonlinear analysis is more complex, but gives the more accurate results. Paper plasticity models are defined in terms of stress and strain. By assuming a linear elastic law, we can define plasticity in terms of strain only. We apply forces in MD and CD directions, so paper material can be stretched and creases appear sharply.

\subsection{Simulation approach for forming the crease lines}

Simulation model for the crease forming is prepared to achieve a local reduction of the bending stiffness and thus simplify the folding operation to make the creases more clearly. We consider paper as a shell surface. Belytchko-Tsay shell element is used for the simulation (Hallquist, 1998). Origami sheet is modeled by the triangular and quadrilateral shell elements within the same material property set.

We have analyzed and evaluated the performance of folding the sheet of paper along the crease line by conducting a little experiment. Considering our human hand as the robot hand, firstly, we fix one side of a folding paper at the crease line and try to bend the other side around the given crease line. The creases do not appear without the force to stretch the paper. In our simulation, we stretch and bend paper at the same time during the folding. In this case the crease lines appear apparently.

With a fiber structure mentioned above, a crease will not appear without external impacts around the crease area. Then, it is not simple procedure to create the crease lines by using paper bending by robot. To fold paper accurately, we suggest 3 states for folding fingers: gripping, rotating, making tensile of paper. These states are not the complex tasks for robotic arms and we expected that after bending, the crease lines appear apparently on paper. Hence, it is necessary to investigate the simulation conditions for gripper placement during the bending operation on paper.

Calculation of a radius of gyration. Several areas, $a_{1}, a_{2}, a_{3}, \ldots$ at distances $y_{1}, y_{2}, y_{3}, \ldots$ from a fixed axis, may be replaced by a single area $A$, where $A=a_{1}+a_{2}+a_{3}+\ldots$ at distance $k$ from the axis, such that $A k^{2}=\sum a y^{2} . k$ is called the radius of gyration of area $A$ around the given axis. Since $A k^{2}=\sum a y^{2}=I$ then the gyration radius is determined: $k=\sqrt{\frac{I}{A}} \quad($ Bird , 2001).

The second moment of area is a quantity much used in the theory of bending of beams. The procedure to determine 
the second moment of area of the regular sections about a given axis is to find the second moment of area of a typical element and to sum all such second moments of area by integrating between appropriate limits. In our case, the second moment of area of the rectangle (a sheet of paper) about the axis (crease line) is found by initially considering an elemental strip of a width $\delta x$, parallel to and a distance x from the crease line. Area of the shaded strip $=b \delta x$. Second moment of area of the shaded strip about the crease line is calculated as $=\left(x^{2}\right)(b \delta x)$.

The second moment of area of the whole folding part about the crease line is obtained by summing all such strips between $x=0$ and $x=l$, i.e. $\sum_{x=0}^{x=l} x^{2} b \delta x$

The second moment of area of the rectangle about the crease line

$$
k=b \int_{0}^{l} x^{2} d x=b\left[\frac{x^{3}}{3}\right]=\frac{b l^{3}}{3} \text {, }
$$

Since the total area of the rectangle, $A=l b$, then $I_{p p}=(l b)\left(\frac{l^{2}}{3}\right)=\frac{A l^{2}}{3}$

$$
I_{p p}=A k_{p p}^{2} \quad \text { thus } \quad k_{p p}=\frac{l^{2}}{3}
$$

$k_{p p}=\sqrt{\frac{l^{2}}{3}}=\frac{l}{\sqrt{3}}$ i.e the radius of gyration about crease line (Fig.6a), where $l$ is the distance from the gripper to the crease line at the initial folding stage.

Current locations for the grippers. The gripper location on a sheet of paper during the folding process is illustrated in Fig. 6b. The grippers are required to be located at the position with a distance $x$ from the rotation axis (crease line) in the next folding stage.

Additionally, it is necessary to ensure avoiding the collisions between the holders and grippers after the rotation of

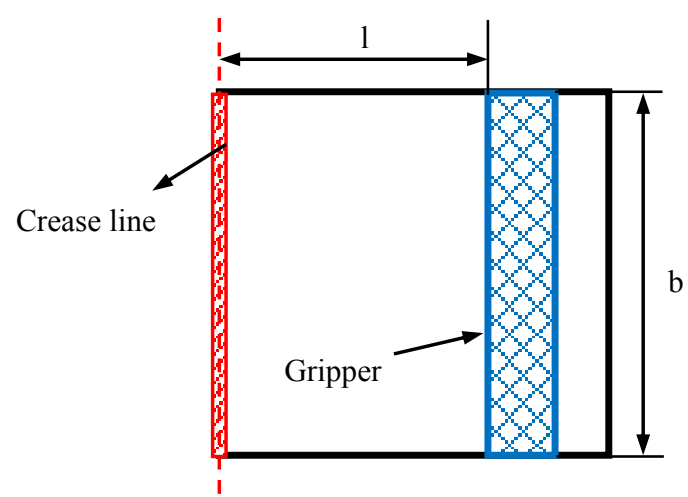

a. Radius of gyration

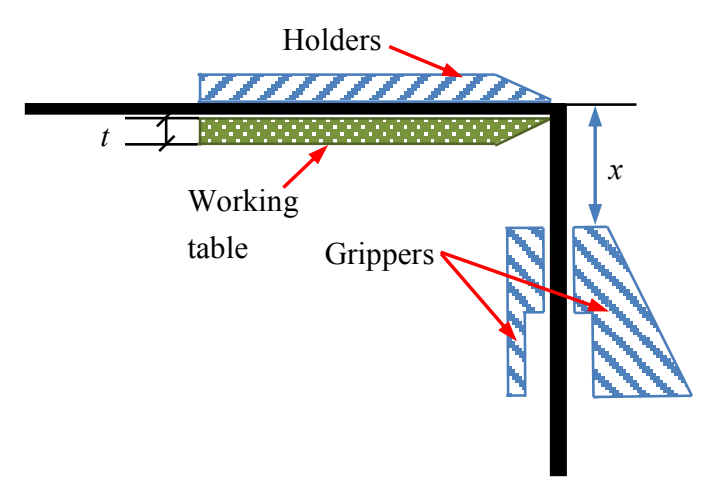

b. Avoiding collisions

Fig. 6 Position for the grippers.

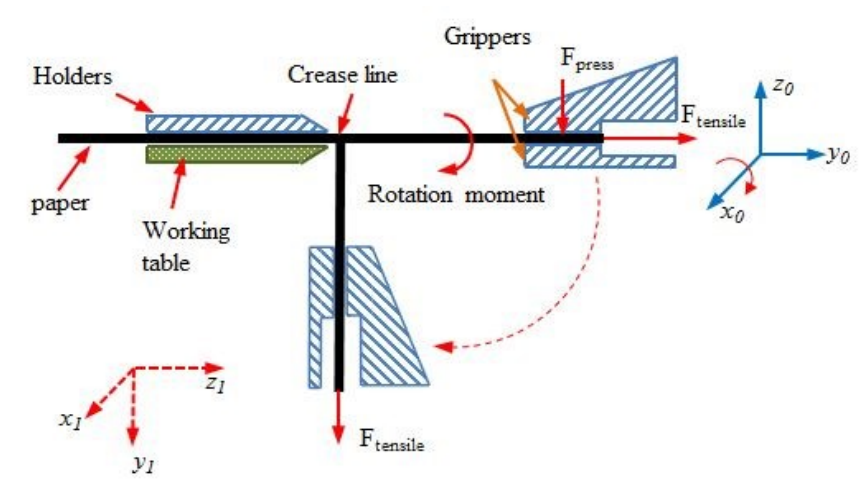

Fig. 7 Sketch of the simulation of the crease forming by the robot fingers. 
a sheet of paper around the crease line by the rotation angle $90^{\circ}$. The thickness of the working table $t$ is calculated according to the gripper location in the current folding stage. The gripper location is determined by the distance $x$ as it is shown in Fig. 6b and should satisfy two conditions: (i) the radius of gyration, (ii) avoiding the collisions.

$$
t<x<\frac{l}{\sqrt{3}},(7)
$$

where $t$ is the thickness of the working table, $\frac{l}{\sqrt{3}}$ is the radius of gyration.

\subsection{Gripper forces}

In this section, we consider the forces that are applied to the paper object by the grippers to produce folding. As in the experiment mentioned above, if a sheet of paper is stretched and bent by human hands at the same time, the creases appear apparently. Then, we decided to imitate this technique in our simulation folding system. Bending alone may not be sufficient to provide the desired shape and stretching (tension) may be required also because paper is made up of vegetable fibers, which are brittle.

When the paper object is grasped by a human hand, the motion of the object is constrained by contacts with the fingers. Hence, the grippers grasp a sheet of paper and pull it. This action is called a transient movement. To prevent being slipped out of the grippers, pressing force is applied on the paper sheet by grippers. Bending with a tensility of the sheet of paper should be performed for forming the crease line. Figure 6 shows the configuration of the movement of the robot hands and applying forces during the folding process. Gripper pressing force $F_{\text {press }}$ is applied in the zdirection and the gripper tensile force $F_{\text {tensile }}$ are applied in the y-direction directions in the initial configuration in a global coordinate system.

In static case, when a sheet of paper does not slip out from the grippers, the minimal value of the pressing force $F_{\text {press }}$ is calculated as below:

$$
F \mu>W \Leftrightarrow F>\frac{m g}{\mu},
$$

where $F$ is a gripper force $F_{\text {press }}, W$ is a weight of a sheet of paper, $\mu$ is a static friction coefficient between the gripper contact portion and the working part of the paper sheet, $m$ is a mass of the sheet, $g$ is the gravitational acceleration. The static friction coefficient is considered in range [0.1-0.2]; therefore the gripping force is usually in 10-20 times larger than the weight of a working object. In our case, the working object is a sheet of paper with a very small weight. For increasing the sharpness of the crease lines, tensile force is applied permanently during bending paper (Fig. 7) in the y - direction in gripper's local coordinate system. The local coordinate system $\left(x_{1} y_{1} z_{1}\right)$ is defined by the rotation of the global coordinate system $\left(x_{0} y_{0} z_{0}\right)$ along the $\mathrm{x}$ - axis. Figure 8 demonstrates accuracy of the crease forming process. Smooth bending is shown in Fig. 8a. As we can see from Fig. 8a, a lack of the tensile force does not allow forming the sharp crease line. Figure $8 \mathrm{~b}$ shows the sharp crease forming after applying the tensile force

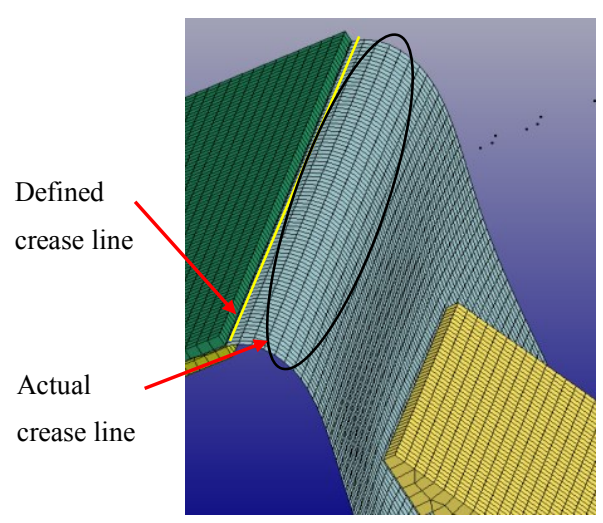

a. Smoothing bending paper with initial coordinates

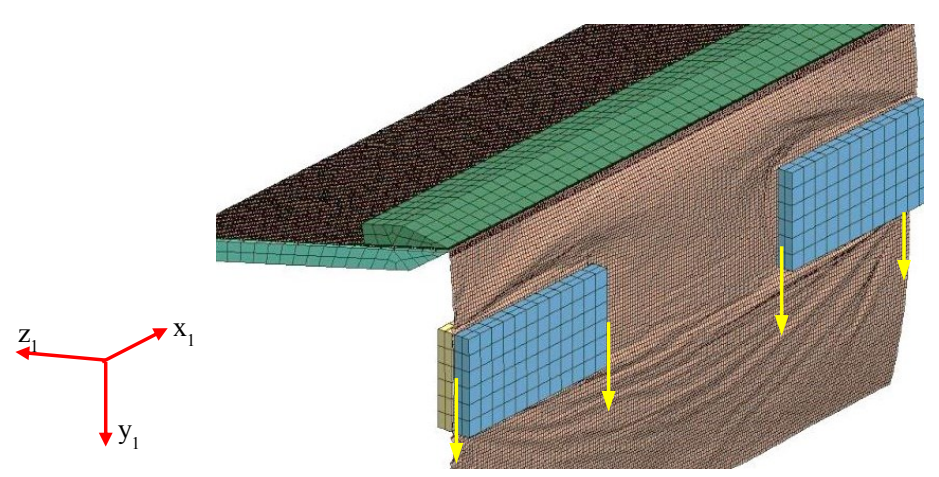

b. Crease appearing after permanent applying the tensile force according to the local coordinates

Fig. 8 Crease forming process. 
permanently during the bending process.

Tensile force is applied along the folding sheet in the direction according to the rotation angle. This is the contact force between the grippers and paper. The contact force is calculated by Eq. (9):

$$
f_{S}=K_{c} \delta
$$

where $K_{c}$ is contact stiffness, $\delta$ is penetration.

\section{Simulation approach \\ 5.1 Origami model}

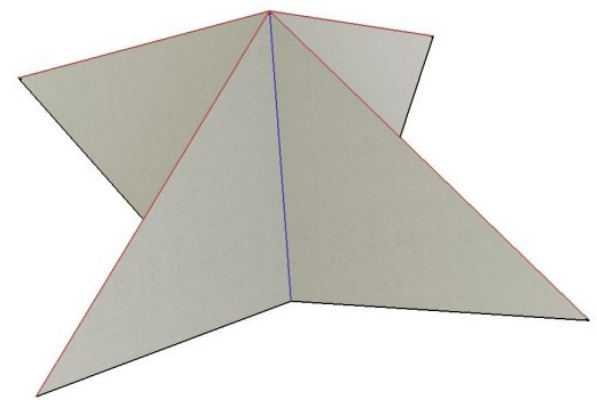

a. The 3D shape

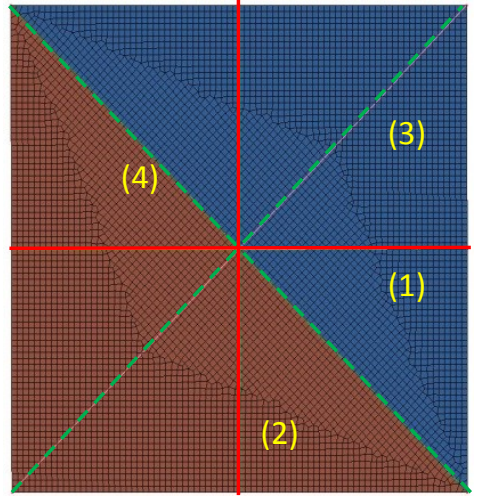

b. The FEM model of the 2D pattern

Fig. 9 The origami model "Star".
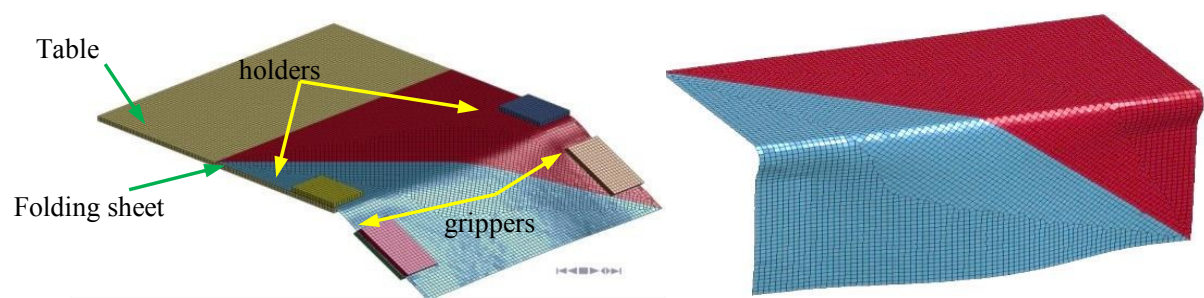

a. Mountain fold
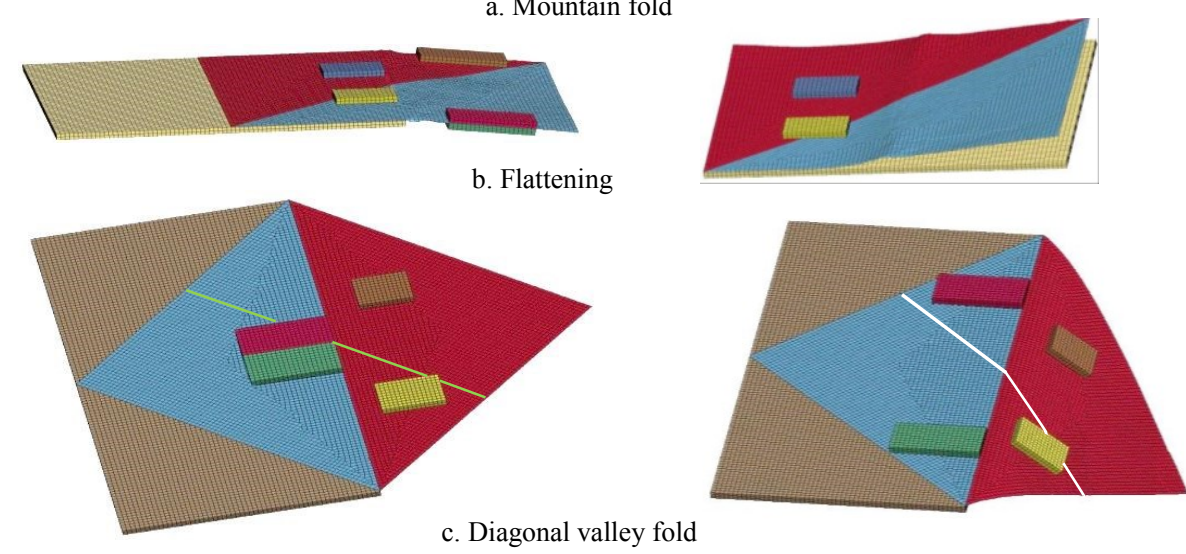

Fig. 10 Folding process.

The 2D pattern of the origami model "Star" (Fig. 9b) was chosen for the simulation of the folding procedure (Fig. 9a). This pattern includes 2 mountain lines and 2 diagonal valley lines.

For the simulation of this model we have to consider the folding sequence to choose the best order for the robot operations. Folding steps are defined by the robot functions: rotation, translation, or flipping the paper sheet.

In the "Star" model, there is one important point on that we have to focus: all crease lines converge at the center of the folding pattern. Hence, the flattening as the robot function should be included as the additional folding step.

We consider 4 main stages in the folding simulation (Fig. 10): 
(1) $90^{\circ}$ bending the paper sheet to make the mountain fold (1) by the folding fingers (Fig. 10a).

(2) The flattening or the translation of the folding sheet to a flat state on the working table by the holders, $90^{\circ}$ rotating the folding sheet and moving it to the folding position (Fig. 10b).

(3) After being flattened on the working table the sheet of paper has to be turned over, rotating by $45^{\circ}$ and moving on the working table to place the crease line at the edge of the working table (Fig. 10c).

(4) Making the mountain fold on the back side of the sheet of paper. In this case, we have the valley fold on the base side of the paper sheet.

These steps should be repeated to make the 2 nd valley fold.

\subsection{FEM - based modeling}

Due to the limitation of the calculation time and the repetition of the operations we have simulated only one mountain and one valley folds in the origami pattern. The numerical simulation of the model in Fig. 9a is produced by using the FEM. In the simulation model we consider only the robot end-effectors and a sheet of paper as the origami 2D pattern.

The grippers and holders are presented as solid models with the hexagonal elements. The size of each element is $0.5 \mathrm{~mm}$. The holders (purple and yellow color in Fig.) constraint the paper sheet model by pressing, while the grippers (red and brown color) bend it around the crease line (Fig. 10a). The upper parts of the grippers are forced to rotate the paper sheet around the crease line up to $90^{\circ}$ or $-90^{\circ}$ from the initial horizontal position according to the valley or mountain lines. Polypropylene (PP) is decided as a material for the grippers and the holders. Plastic material provides decreasing the weights of the robotic parts.

"MAT54/55-Enhanced composite damage" material is chosen as paper material in LS-DYNA simulation, because the mechanical properties of this material are closed to paper. A sheet of paper is described as a shell structure with the mixed elements (triangular and quadrilateral) with a size of $5 \mathrm{~mm}$ (Liedberg, 2014). Using the small mesh element's size allows us to receive the accurate calculation results. In this simulation, the thickness of a sheet of paper is decided as $0.1 \mathrm{~mm}$ (for the ordinary paper thickness is measured in the range: $0.08-0.1 \mathrm{~mm}$ ). Figure $9 \mathrm{~b}$ shows the mesh of the paper structure. In the given origami model, there are two crease lines in the diagonal directions. In this case, the simulation may have difficulty for forming the creases. For solving this problem, the simulation model of the origami paper pattern is presented by a combination of two triangle parts separated by the diagonal. Meshes of these two parts are connected by merging the duplicate nodes between them. The total number of mesh elements in the full simulation model is 22003 .

The grippers are set up as above mentioned method and the holders are placed in the crease area. The distance from the grippers to the crease lines is calculated by Eq. (7) and has to satisfy Eq.(10):

$$
10<x<200 / \sqrt{3}(\mathrm{~mm})
$$

The length of the mountain fold is $200 \mathrm{~mm}$, the thickness of the holders is $10 \mathrm{~mm}$.

In this case, we choose the location $x=50 \mathrm{~mm}$ for the grippers. The gripping force, which is used in this simulation, is the human finger pressing force of $40 \mathrm{~N}$.

\section{Discussion of the simulation results \\ 6.1 Result of the crease forming}

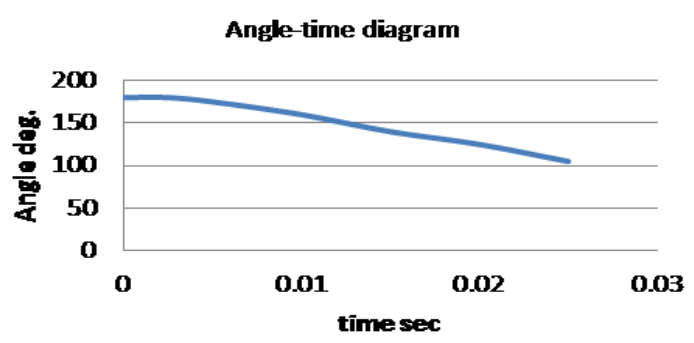

a. Bending angle with respect to time

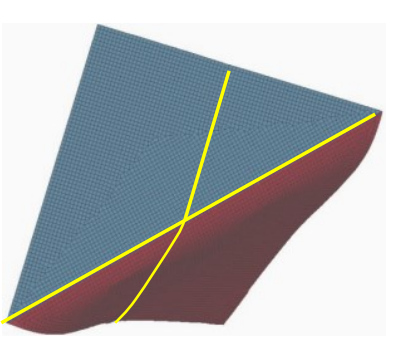

b. Shape of paper after folding

Fig. 11 Simulation results. 


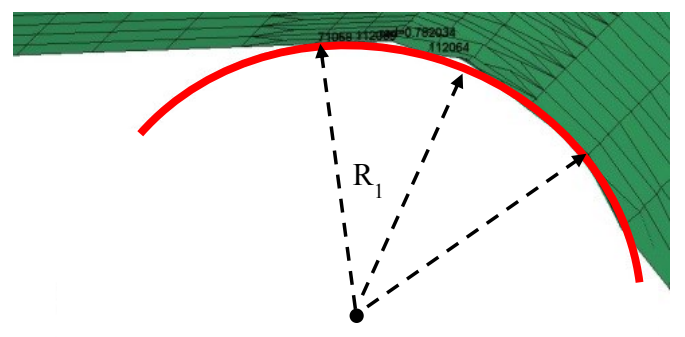

a. Before applying tensile force

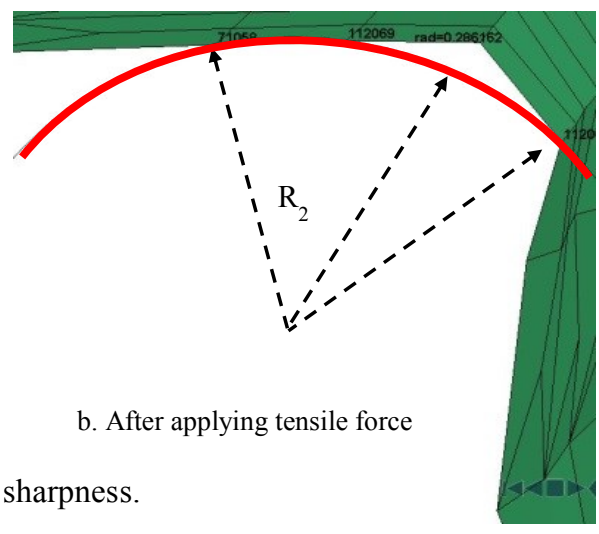

Fig. 12 Illustration of the crease sharpness.

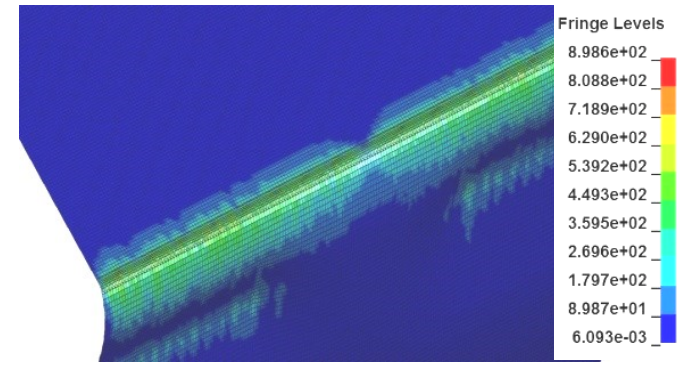

a. After bending

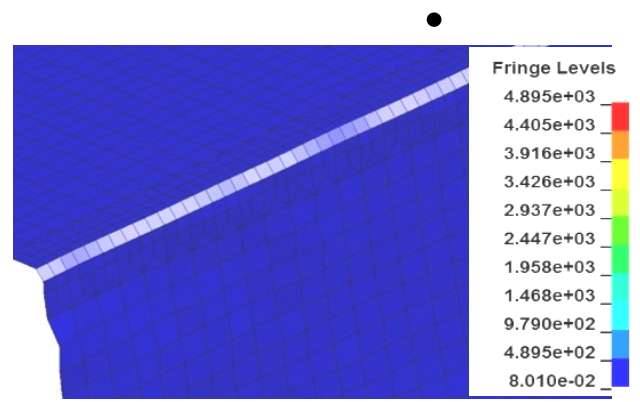

b. After flattening during a bending

Fig. 13 Von-Mises stress concentration on folding paper.

3 processing phases: bending, translating, and flattening are under our consideration. We judge the performance of the system by the crease's angles and the deformation of paper during the process.

The most important result is the appearance of a crease line. We have to check that it appears apparently or not by the change of the dihedral angle between two sides of the paper sheet. From Fig. 11a we can see that during the producing paper folding by the grippers around the crease line by $90^{\circ}$, the dihedral angle between a sheet part on the working table and the folded sheet part is measured as $107^{\circ}$ that is closed to $90^{\circ}$. It means a sharp crease is formed. After that, the paper material remembers the crease line. In Fig. 11b, we demonstrate the crease lines after applying bending. To estimate the accuracy of the folding operation we apply circle spline interpolation. Circle is used to create closed circular splines made of three mesh nodes. In Fig. 12 we illustrate this procedure. In the result of the rotation of the sheet of paper around the designed crease (Fig. 12a), the crease does not appear, the bump between two sides of the sheet is visual. All of three mesh nodes out of the crease area and a node on the generated crease are located on the circle with radius $R_{l}$. In the case of applying a tensile force with the combination with rotation, two mesh nodes out of the crease area are located on the circle with radius $R_{2}$, but a crease node does not belong to the circle. This approach shows the accurate sharpness of forming the creases (Fig. 12b). Another way to prove the appearance of the crease line on a paper sheet is the results of finite element stress analysis. In FEM, computer is unable to distinguish between a ductile and a brittle material. We use the Von-Mises criteria for checking failure in structures, regardless the applicable theory for the material (Budynas, 1999). As paper is made of brittle material, values of Von-Mises stress are greater in the crease than in others (Fig. 13). This means in this area, structure of paper is damaged, and the crease is formed sharply.

Additionally, the appearance of the crease is illustrated through the stress-strain diagram. As paper is considered as a brittle material, we cannot find the yield point. For analysis, we use a quadrilateral sheet of paper with the geometrical size $40 \times 40(\mathrm{~mm})$ and the thickness of $0.1 \mathrm{~mm}$. We model paper as elastic one, when a crease appears, the paper structure is broken. The stress-strain diagram in MD-CD ( $\mathrm{x}, \mathrm{y})$ direction is given in Fig. 14 for forming the mountain crease (the model "Star"). Because of noisy data, we consider the diagram as 2 parts to approximate a linear curve. As one can see from Fig. 14d and Fig.14e the crease are formed at the moment of $0.015(\mathrm{~s})$ when strain $\varepsilon=0.2$ and stress $\sigma=178\left(\mathrm{~N} / \mathrm{mm}^{2}\right)$. After crease forming, the stress-strain curve shows nonlinear behavior, it means the crease exists. 


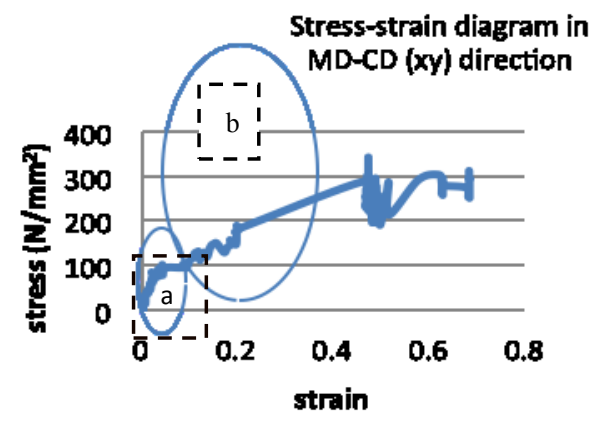

a. Stress-strain diagram

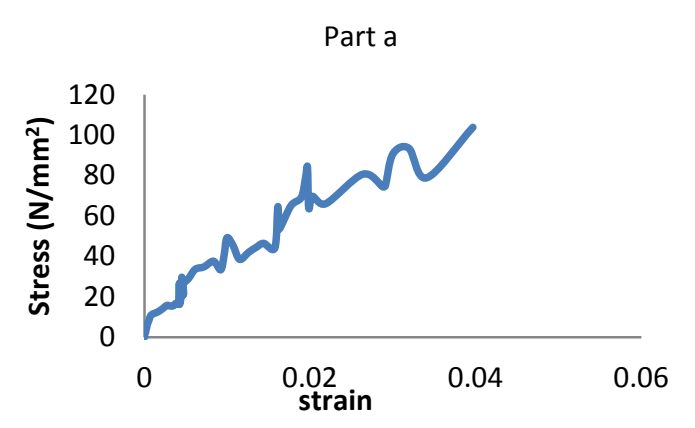

b. Stress-strain diagram part 1

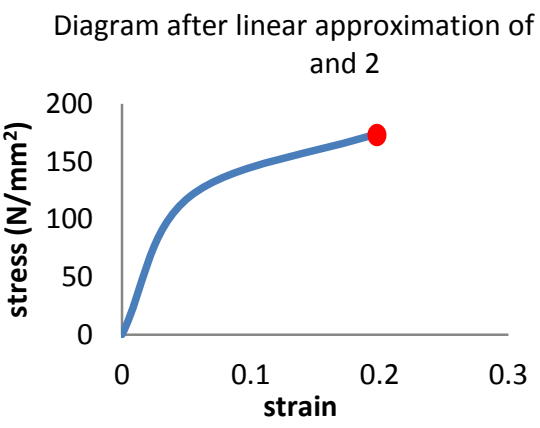

d. Stress-strain diagram after linear approximation

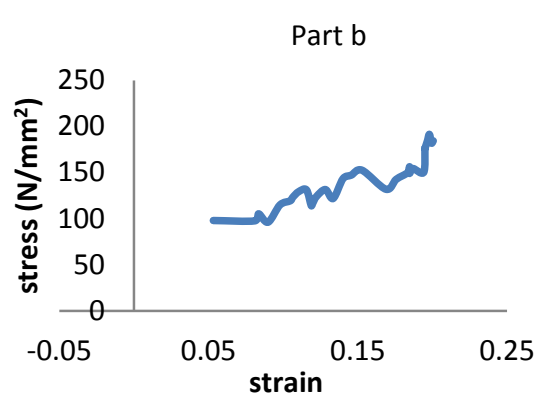

c. Stress-strain diagram part 2

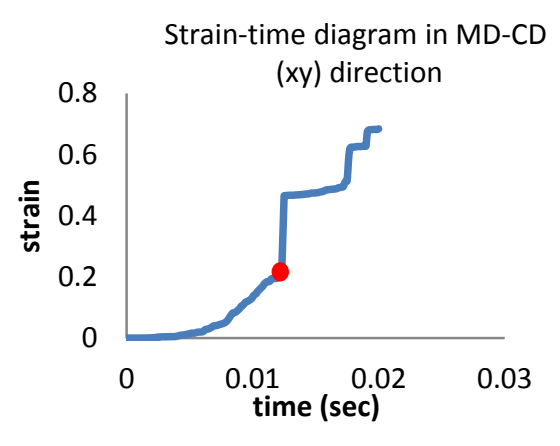

e. Strain with respect to time

Fig. 14 Elastic stress-strain analysis.

\subsection{Motion planning for the robot arm}

We have decided the motion of the robotic arms based on a simulation of the folding sequence for the given model (Fig. 15) such as:

1. Start position is the mountain fold (1-1). Positions of the grippers and the holders are decided according to the conditions described in Section 5.2. The grippers produce up $90^{\circ}$ bending the sheet of paper along the crease line by applying tensile forces in MD and CD directions. The holders fix the sheet on the working table under the pressing force.

2. After forming a crease line, translating and flattening the sheet are produced by the holders. The working table is rotated to the new start position; the grippers and the holders are located for bending mountain fold (2-2) with the same conditions as shown in the step 1.

(Repeat 2 beyond steps to make the second fold)

3. Flattening the sheet on the working table; turning the folding paper over by the grippers; rotating the working 
table up $45^{\circ}$; moving the valley fold (3-3) to the crease position by the holders.

4. Making the mountain fold on the back side of the sheet of paper by the grippers. In this case, we have the valley fold on the main side of the sheet.

5. Flattening the folding sheet on the working table by the holders; rotating the working table of $90^{\circ}$ and repeating the step 4 to make the valley fold (4-4) by the grippers.

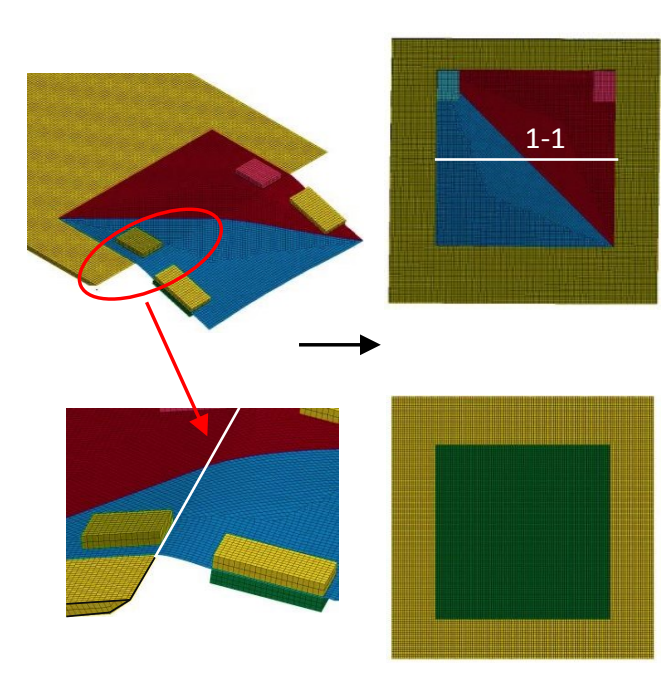

Step 1

Step 2

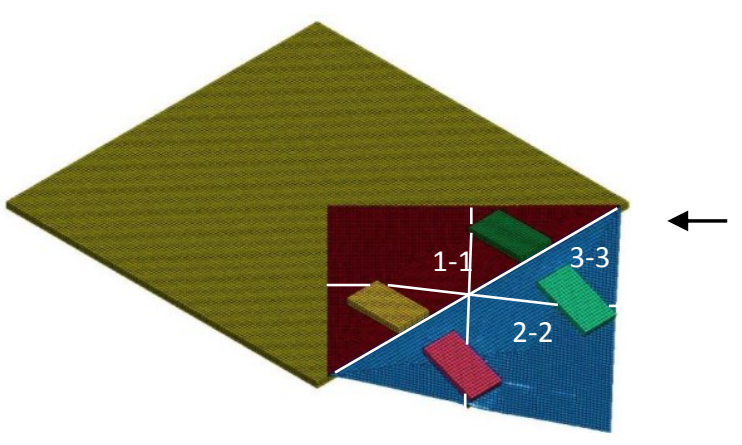

Step 5

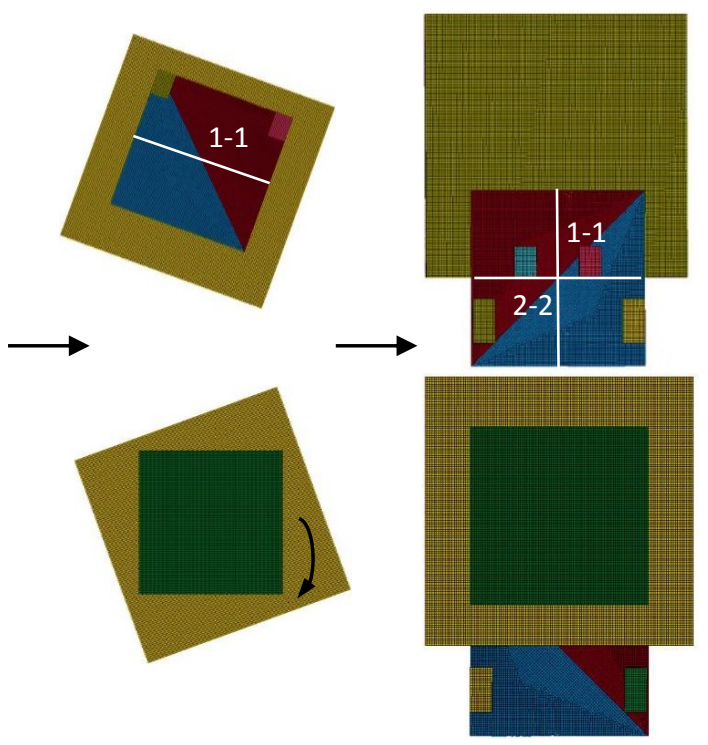

$2^{\text {nd }}$ mountain fold
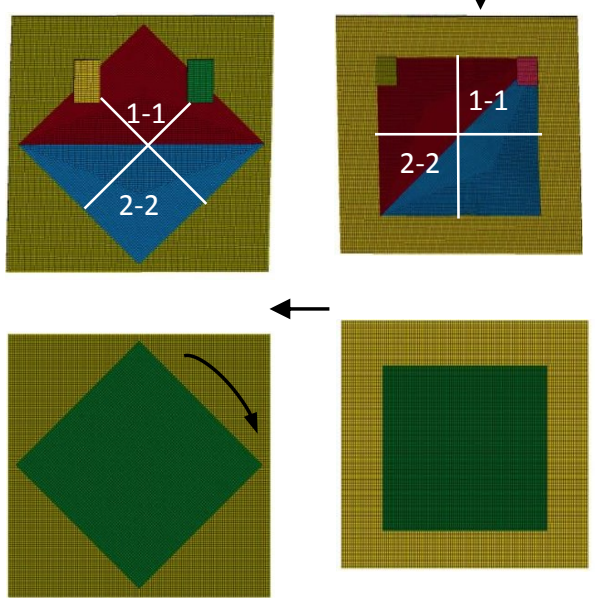

Step 4

\subsection{The Design Developing Stage}

Fig. 15 Folding sequence for the robot motion.

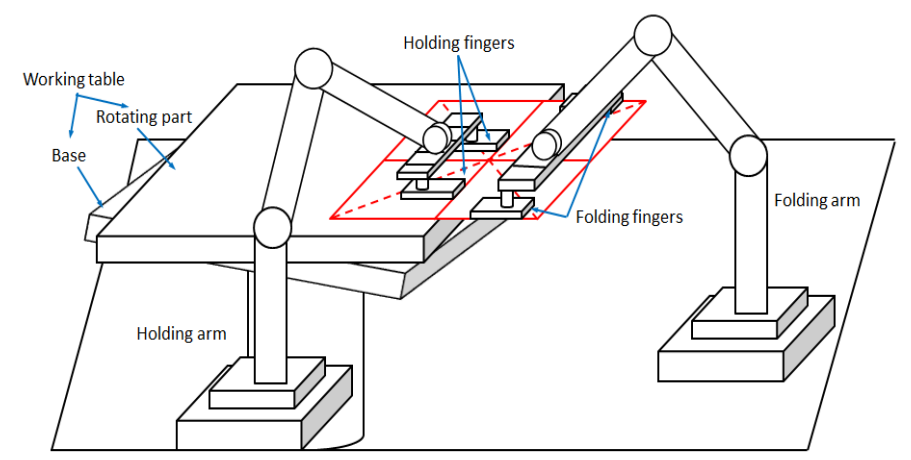

Fig. 16 Sketch of the simulation-based final design of the robot. 


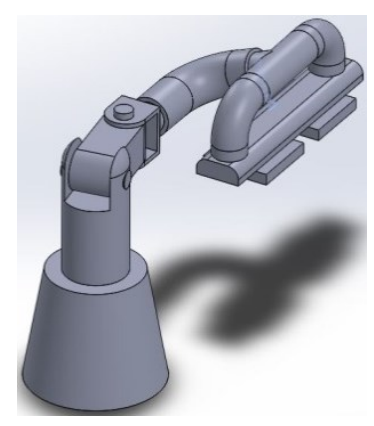

a. Holding arm

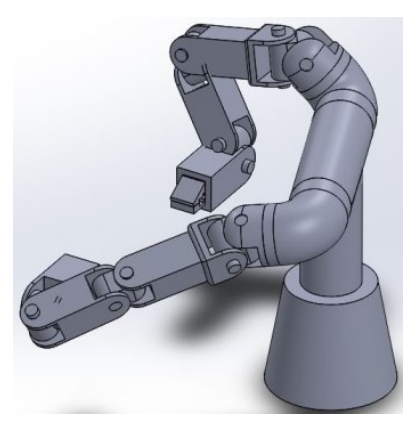

b. Folding arm

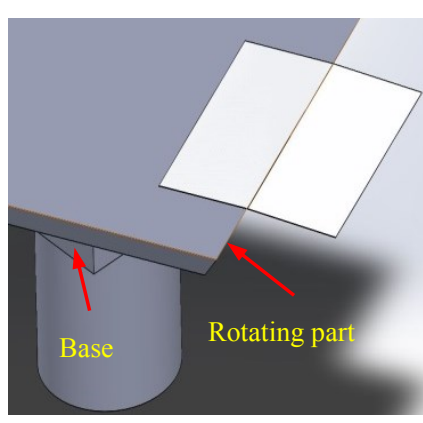

c. Working table

Fig. 17 Configuration of the robot (CAD models).

The realistic robot design meets requirements such as mechanical, geometrical, topological. During the simulation of crease forming, some problems are probably determined, for instance: paper wrinkling and tearing, no forming the creases, no possibilities to change a sheet's position on the working table for producing the next folding.

A stage subsequent to schematic design, where the schematic design decisions are worked out in greater detail, we call the Design Developing Stage. The Design Developing Stage includes the modification of the Schematic Design. According to the problems formulated above, we have made the simulation solution, such as:

(1) Paper wrinkling and tearing: by editing the grippers that can move from the center of the crease line in the direction to its ends in order to exclude wrinkles on paper and strong fix paper strongly on the working table;

(2) Making crease: by the force values and directions; the locations of the grippers.

We modify the conceptual robot design for forming the crease lines, which satisfies the origami pattern. The modified design is different from the schematic design by changing the number and shapes of both holders and grippers. The holders will be able to move along the crease line by the screw mechanism, which is included in the design system. The grippers are placed at the edges of the sheet of paper instead of one single folding finger located along the whole crease lines as shown in Fig. 1. Additionally, we change the space positions of these two arms and create working table with two parts - a base and a rotating part - to make it easier to place the origami sheet of paper at the current position for folding. Figure 16 is the illustration of the Design Developing Stage. Table 1 demonstrates the difference between the schematic and modified robot designs. Design Developing Stage can be repeated several times according to the simulation results for making a good design decision. After the modification, the final design is more complicated than the schematic one. It means that the complex origami structures cannot be folded by a simple robot system.

The configuration of the robot arms with 3 links and universal joints to connect robot links and the working table design are illustrated in Fig. 17. Universal joints allow links to bend in every direction, so our robot is flexible.

\subsection{The reachability of the robot manipulators}

In this research, A4 paper format is the largest object for folding operation; therefore it is necessary to consider the kinematic problems to ensure that the reachable region of this robot is enough (Thai, 2012).

The kinematic equations of the robotic system for one arm are as below:

$$
\begin{aligned}
& f_{1}=l_{1} \cos q_{1}+l_{2} \cos \left(q_{1}+q_{2}\right)+l_{3} \cos \left(q_{1}+q_{2}+q_{3}\right) \\
& f_{2}=l_{1} \sin q_{1}+l_{2} \sin \left(q_{1}+q_{2}\right)+l_{3} \sin \left(q_{1}+q_{2}+q_{3}\right), \\
& f_{3}=q_{3}
\end{aligned},
$$

where $\left[q_{1}, q_{2}, q_{3}\right]$ are the joint angles, $\left[l_{1}, l_{2}, l_{3}\right]$ are the link' lengths of the robot arm. To exclude the singularity we adopted the objective function for optimization the reachable region is obtained by using the Jacobian matrix as follows:

$$
\phi(\boldsymbol{q})=\sqrt{\operatorname{det}\left[\boldsymbol{J}(\boldsymbol{q}) \boldsymbol{J}^{T}(\boldsymbol{q})\right]},
$$


When the function $\phi(q)$ reaches the extreme value, the robot arm avoids singularity.

The end-effector is considered to move along a circle during the folding process. In this research, we design our robot for folding A4 paper; the motion of the robot arm is calculated by MATLAB and is illustrated in Fig. 18. The configuration of the robotic arm that is satisfied to the calculated reachability is given in Table 2.

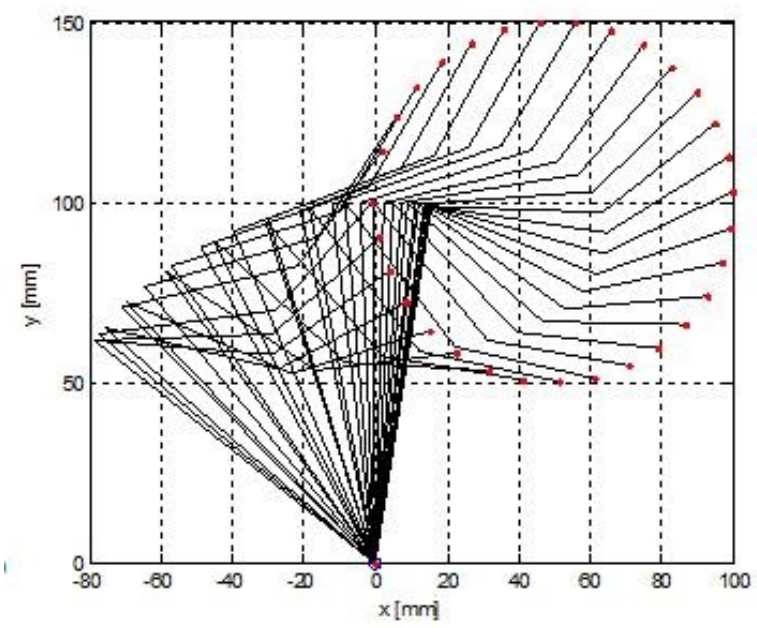

Fig. 18 MATLAB calculation results of the robot arm motion in the 2D space.

Table 1 Differences between schematic and modified designs.

\begin{tabular}{c|c|c}
\hline \hline & Schematic Design & Modified Design \\
\hline Number of grippers & 1 & 2 \\
\hline Number of holders & 1 & At the edge of the sheet of paper \\
\hline Gripper position & not defined & Holders: translating along the crease lines \\
\hline \multirow{2}{*}{ Robot arm' action } & Holders: fixing paper along the \\
& whole crease line & Grippers: tensile and rotation \\
\cline { 2 - 3 } & Grippers: rotation & 2 parts: base and rotating part \\
\hline Working table & 1 part: base &
\end{tabular}

Table 2 Robot arm's geometry and topology.

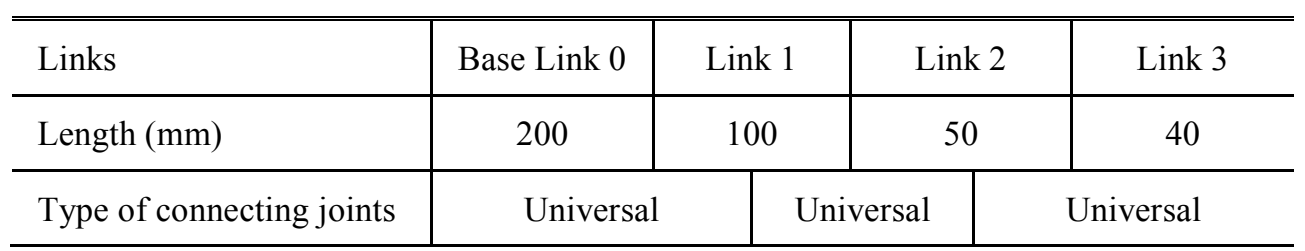




\subsection{Discussions and limitations of the origami-performing robot 6.5.1. Hardware designing}

In this research, with the purpose of home-based using, our robot system is designed for folding A4 paper format. The geometrical and topological parameters of the robot arms are calculated for this type of paper format according to the simulation results by LS-DYNA software as shown in Fig. 17 and Table 2.

We designed the edges of the working table as a blade shapes according to the folding operations: we fix one part of q sheet of paper on the working table along the crease line (the working edge of the working table) and bend the free part of the sheet around the crease line (the working edge) see Fig.17c. In this case, a sheet of paper can be bended more than $90^{\circ}$. That action and the movable holders allow us to form the accurate crease lines. The holders are designed to be movable for fixing a sheet of paper on the working table and sharpening the crease lines. For the grippers the "crank-slider" mechanism is chosen. The grippers, which functions are grasping and bending the sheet of paper, are designed according to this mechanism and developed by the CAD software "SolidWorks".

\subsubsection{The ability to perform the various origami structures}

The presented robot system can perform origami models without mechanical redesigning for each new origami folding pattern, but we have to decide the planning of the robotic arm motion according to the folding order. That is connected to the calculation of the values of the rotation angles of the working table and the locations of the grippers for forming each crease line. By this reason, for the current robot design, we can consider the types of the origami models, such as: cardboard packaging and the artificial formed free shapes. The folding lines in the packaging pattern are mostly fully located across a sheet of paper. This type includes the crease lines that do not influence to others, therefore the robot arms can produce one by one bending, rotating, and translating operations that are necessary for forming the crease lines. In the case of the artificial formed free shape, more actions (operations) of the robot arms are required for forming the creases. The reason is that the valley and mountain lines can be intersected for building the 3D concave shape. We use the "Star" model as an example of the artificial formed free shape. The robot operations for folding the "Star" model are: bending, rotating, translating, and turning a sheet of paper over. For confirming the ability to perform another origami model we consider the "tadpole" model that is the target of (Tanaka, 2008). Folding sequence of the robot operations, which is developed manually, is shown in Fig. 19. After all, we can say that the suggested robotic system is ready to perform the "tadpole" model without redesigning of the robot configuration, but with reprogramming of the robot arm motion.

\subsubsection{Limitation of the current study}

There are some limitations in our methodological approach for forming the creases. Currently, the robot is not able to form origami patterns with line segments like "zig-zag" fold (the "Miura-ori" model). With this kind of the folding structure we need to consider the best solution for forming crease lines without redesigning our robot system.

Additionally, in the simulation of a whole motion of the robot, some limitations and the additional mechanical problems may be encountered, such as: robot's workspace for determining the volume, in which the robot's endeffector may act, limitations of joints, singularity, collisions between the robot arms. These kinds of limitations and others cannot be determined in the current simulation study. In next stage of the research, we should consider a simulation of the whole robot motion to solve the mechanical problems and to make the perfect design decision. In this research, we did not consider the robot software, for instance, the recognition of the origami patterns. It can be our future research: to study a software for a realistic design of the proposed robotic system. 


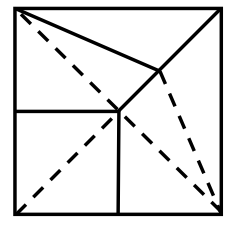

2D pattern

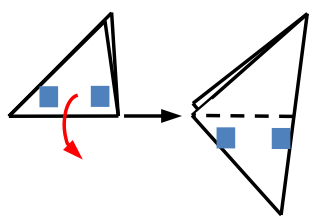

Step 3: Valley fold

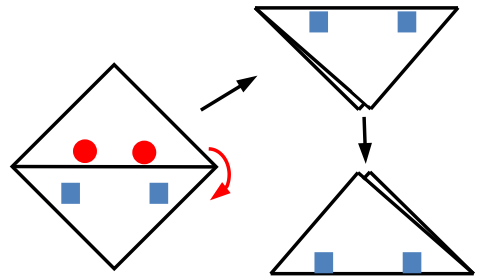

Step 1: Mountain fold + rotate pattern

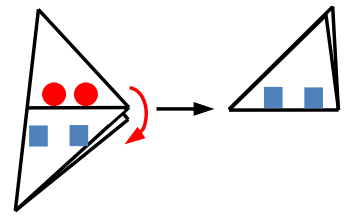

Step 2: Mountain fold
: Holder $\quad$ : Gripper

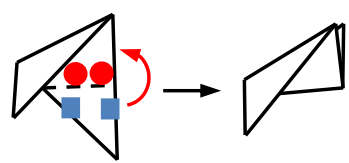

Step 5: Valley fold

Fig. 19 The "tadpole" model: folding sequence.

\section{Conclusion}

In this paper, we demonstrate an approach for designing the origami-performing robot based on the simulation of forming the crease lines in the origami 2D patterns by the robot end-effectors. This method is proven to be useful by numerical simulations by using the FEM on LS-DYNA software. The robot design approach includes 2 main stages: Conceptual (schematic) and Design developing. Based on the conceptual robot design, we simulate forming the sharp creases on a sheet of paper with thickness of $0.1 \mathrm{~mm}$ by bending and stretching paper for defining the gripper's placement on a sheet of paper, pressing and tensile forces. In the Design developing stage the final robot's hardware is decided according to the simulation results. The proposed design method has the main advantages of providing a large coverage of the design solutions according to the main requirements for the production of the origami models. We show that the problems of the creases forming on a sheet of paper by the robot arms can be solved by a simulation only without a series of experiments.

Currently, packaging and the origami models with the formed free shapes are suitable for the presented robot system. The free shape origami model "Star" with the ordinary paper material and various types of the crease lines, including the diagonal one, is chosen as an experimental model for demonstration of the suggested approach.

In the current research, the robot design is based on using A4 paper format and includes 3 main parts: 2 robot arms and a working table. The final design is more complicated than the schematic design, but complex origami models can be produced by reprogramming the robot arm motion and without redesigning. The calculated geometrical sizes of the compact robot allow us to use it in limited spaces such as home room (for instance, on a desk), children facilities, offices and so on for the production of paper models.

In the next stages, the specific design for each robot component should be considered to make a complete design with controlling and programming to operate the robotic system. The simulation of the final design of the origami-performing robot should be done also. It is necessary to test our simulation-based design approach by producing a real prototype of the robot, which includes both hardware and software parts. In the case of the positive test results, the robotic system can be suggested for manufacturing.

The real origami-performing robot system can find implementation in many areas of applications such as: engineering, papercraft, architecture, furniture. 


\section{References}

Balkcom, D.J, Mason, M. T, Introducing Robotic Origami Folding, In Proc. IEEE International Conference on Robotics and Automation, (2004), pp. 3245-3250.

Bird J., Newnes Engineering Mathematics, Pocket book, Oxford, (2001), pp.346-347.

Budynas, R.G, Advanced Strength and Applied Stress Analysis, Text book, $2^{\text {nd }}$ Edition, McGrawhill, (1999).

Elbrechter, C., Haschke, R., Ritter, H., Folding paper with anthropomorphic robot hands using real-time physics-based modelling, Proc. IEEE-RAS International Conference on Humanoid Robots, (2012), pp. 210-215.

Hallquist,J.O, Belytchko-Liu-Tsay shell, LS-DYNA Theoretical Manual, Livermore Software Technology Corporation, (1998), pp. 6.1-6.12.

Liedberg M., Crash behavior of composite structures, Master thesis, (2014).

Lister, D., What makes paper crease, www.british.com.info/academic/lister/what_makes_paper_crease.php

Livermore Software Technology Corporation, LS-DYNA Keyword User's manual-Volume II, version 960, (2001).

Namiki A. and Yokosawa Sh., Robotic Origami Folding with Dynamic Motion Primitieves, Proc. in IEEE/RSJ International Conference on Intelligent Robots and Systems (IROS), (2015), pp. 5523-5628.

Tanaka, K., Kamotani, Y., Yokokohj, Y., Origami Folding by a Robotic Hand, In Proc. in IEEE/RSJ International Conference on Intelligent Robots and Systems, (2007), pp. 2540-2547.

Thai P.T, The inverse kinematics problem under consideration of dynamic calculation, Master thesis (in Vietnamese) (2012).

Yao, W., Dai, J.S, Medland, T., Mullineux, G., A reconfigurable robotic folding system for confectionery industry, Industrial Robot: An International Journal, Vol. 37 Iss 6, (2010), pp. 542-551. 